\title{
THE
}

\section{Language Impairment in Children Perinatally Infected with HIV Compared to Children Who Were HIV-Exposed and Uninfected}

\author{
Mabel L. Rice \\ L. Buchanan \\ University of Rhode Island, buchanan@uri.edu \\ George K. Siberry \\ Kathleen M. Malee \\ Bret Zeldow
}

See next page for additional authors

Follow this and additional works at: https://digitalcommons.uri.edu/php_facpubs

This is a pre-publication author manuscript of the final, published article.

Terms of Use

All rights reserved under copyright.

\section{Citation/Publisher Attribution}

Rice, M. L., Buchanan, A. L., Siberry, G. K., Malee, K. M., Zeldow, B., Frederick, T.,...Williams, P. L. (2012). Language Impairment in Children Perinatally Infected with HIV Compared to Children Who Were HIVExposed and Uninfected. Journal of Developmental \& Behavioral Pediatrics, 33(2), 112-123. doi: 10.1097/ DBP.0b013e318241ed23

Available at: http://dx.doi.org/10.1097/DBP.0b013e318241ed23

This Article is brought to you for free and open access by the Pharmacy Practice at DigitalCommons@URI. It has been accepted for inclusion in Pharmacy Practice Faculty Publications by an authorized administrator of DigitalCommons@URI. For more information, please contact digitalcommons-group@uri.edu. 


\section{Authors}

Mabel L. Rice, L. Buchanan, George K. Siberry, Kathleen M. Malee, Bret Zeldow, Toni Frederick, Murli U.

Purswani, Howard J. Hoffman, Patricia A. Sirois, Renee Smith, Peter Torre III, Susannah M. Allison, and

Paige L. Williams 


\title{
Language Impairment in Children Perinatally Infected with HIV Compared to Children Who Were HIV-Exposed and Uninfected
}

\author{
Mabel L. Rice, PhD ${ }^{1}$, Ashley L. Buchanan, $\mathrm{MS}^{2}$, George K Siberry, MD, MPH ${ }^{3}$, Kathleen M. \\ Malee, $\mathrm{PhD}^{4}$, Bret Zeldow, MS${ }^{2}$, Toni Frederick, PhD, MSPH ${ }^{5}$, Murli U. Purswani, MD ${ }^{6}$, \\ Howard J. Hoffman, MA ${ }^{7}$, Patricia A. Sirois, PhD $^{8}$, Renee Smith, PhD $^{9}$, Peter Torre III, PhD, \\ MS ${ }^{10}$, Susannah M Allison, PhD $^{11}$, and Paige L. Williams, PhD $^{2}$ for the Pediatric HIV/AIDS \\ Cohort Study (PHACS)
}

${ }^{1}$ Speech, Language, Hearing Department, University of Kansas, Lawrence, KS ${ }^{2}$ Center for Biostatistics, Harvard School of Public Health, Boston, MA ${ }^{3}$ Pediatric Adolescent Maternal AIDS Branch, Eunice Kennedy Shriver National Institute of Child Health and Human Development, National Institutes of Health, Bethesda, MD ${ }^{4}$ Northwestern University Feinberg School of Medicine, Children's Memorial Hospital, Chicago, IL ${ }^{5}$ Maternal, Child and Adolescent Program for Infectious Diseases and Virology, Dept. of Research Pediatrics, USC Keck School of Medicine, Los Angeles, California ${ }^{6}$ Albert Einstein College of Medicine, Division of Pediatric Infectious Disease Department of Pediatrics Bronx-Lebanon Hospital Center Bronx, NY ${ }^{7}$ Epidemiology and Statistics Program, Division of Scientific Programs, National Institute on Deafness and Other Communication Disorders (NIDCD), National Institutes of Health, Bethesda, Maryland ${ }^{8}$ Department of Pediatrics, Tulane University School of Medicine, New Orleans, LA ${ }^{9}$ Department of Pediatrics, University of Illinois at Chicago, Chicago, IL ${ }^{10}$ School of Speech, Language, and Hearing Sciences, San Diego State University, San Diego, CA ${ }^{11}$ Center for Mental Health Research on AIDS, National Institute of Mental Health (NIMH), National Institutes of Health, Bethesda, MD

\section{Abstract}

Objective-To investigate risk for language impairment in children perinatally infected or exposed to HIV.

\begin{abstract}
Methods-We evaluated the prevalence of language impairment (LI) in 7-16 year old children with perinatal HIV infection (HIV+) compared to children HIV-exposed and uninfected (HEU), using a comprehensive standardized language test (CELF-4). LI was classified as primary LI (PriLI) (monolingual English exposure and no cognitive or hearing impairment), concurrent LI (ConLI) (cognitive or hearing impairment), or no LI. Associations of demographic, caregiver, HIV disease and antiretroviral treatment (ART) factors with LI category were evaluated using univariate and multivariable logistic regression models.
\end{abstract}

\begin{abstract}
Results-Of 468 children with language assessments, 184 (39\%) had LI. No difference was observed by HIV infection status for overall LI or for Pri-LI or Con-LI; mean (SD) CELF-4 scores were 88.5 (18.4) for HIV+ vs 87.5 (17.9) for HEU. After adjustment, Black children had higher odds of Pri-LI vs no LI ( $\mathrm{aOR}=2.43, p=0.03)$. Children who were Black, Hispanic, had a caregiver
\end{abstract}

Corresponding Author: Mabel L. Rice, PhD, 1000 Sunnyside Avenue, 3031 Dole Human Development Center, University of Kansas, Lawrence, KS 66045, mabel@ku.edu, Telephone: 785-864-4570, Fax: 785-864-4571.

Publisher's Disclaimer: This is a PDF file of an unedited manuscript that has been accepted for publication. As a service to our customers we are providing this early version of the manuscript. The manuscript will undergo copyediting, typesetting, and review of the resulting proof before it is published in its final citable form. Please note that during the production process errors may be discovered which could affect the content, and all legal disclaimers that apply to the journal pertain. 
with low education or low IQ, or a non-biological parent as caregiver had higher odds of Con-LI vs no LI. Among HIV+ children, viral load $>400$ copies $/ \mathrm{ml}$ (aOR=3.04, $p<0.001$ ), CDC Class C $(\mathrm{aOR}=2.19, p=0.02)$ and ART initiation $<6$ months of age $(\mathrm{aOR}=2.12, p=0.02)$ were associated with higher odds of Con-LI vs. no LI.

Conclusions-Children perinatally exposed to HIV are at high risk for LI, but such risk was not increased for youth with HIV. Risk factors differed for Pri-LI and Con-LI.

\section{Keywords}

Pediatric HIV infection; language impairment; antiretroviral therapy

\section{INTRODUCTION}

Children perinatally-infected with Human Immunodeficiency Virus (HIV) are at risk for impairments in cognitive functioning ${ }^{1-2}$. Increased risk for language impairment is also reported, which can affect academic performance or adherence to medication ${ }^{3-6}$. Impaired verbal functioning has been shown to be associated with HIV disease progression ${ }^{7}$ and greater immunosuppression ${ }^{8}$. In a sample of children infected with HIV who were evaluated prior to the availability of antiretroviral (ARV) drug therapy, the prevalence of language impairment (LI) was $10 \%{ }^{9}$. Treatment with one or two nucleoside reverse transcriptase inhibitors (NRTIs) in the pre-HAART (highly active antiretroviral therapy) era has been associated with improvement, though not normalization, of overall cognitive and specific language measures in some studies ${ }^{5,10}$. However, Wolters et al found that over the course of a 24-month ART regimen, cognitive abilities remained stable while language abilities continued to decline, suggesting differential effects on specific brain functions ${ }^{8}$. Initiation of HAART has been associated with stabilization in measures of overall cognitive and language function ${ }^{11-14}$. However, studies focused on assessment of language function in school-aged children in the HAART era are lacking.

It is not clear if language impairments in children with HIV infection are indications of a global developmental impairment, including non-verbal and verbal intelligence, or whether language impairment can be a selective deficit. Another possible source of language impairment is related to risk for hearing loss, possibly as a consequence of mitochondrial dysfunction. Prior studies suggest that HIV and its treatment are associated with mitochondrial dysfunction ${ }^{15}$ and it has been demonstrated that mitochondrial dysfunction/ mutation is associated with sensorineural hearing $\operatorname{loss}^{16-17}$.

In healthy children without HIV exposure, language impairments appear in children whose general cognitive abilities are well within or even above age expectations. The condition of Specific Language Impairment (SLI) is defined as language impairments without other developmental impairments. In a study of 7,218 kindergarten children to determine the prevalence of SLI, Tomblin et $\mathrm{al}^{18}$ reported $7.4 \%$ with SLI and $12 \%$ of the children with language sparing, children who scored low on nonverbal IQ assessment (in the range of 7087) but demonstrated language performance within normal limits ${ }^{19}$, suggesting a dissociation of language acquisition and nonverbal intelligence. Similarly, Rice et al ${ }^{20}$ documented that children with low nonverbal IQ levels can have grammatical skills within or above typical levels. On the other hand, children who have both language impairments and nonverbal cognitive impairments scored lower, and grew more slowly in language skills between 6 and 10 years, than children with SLI. In effect, language impairments are not necessarily part of a global developmental impairment in healthy children but if both language and cognition are low, the combination yields lower language performance than language impairment alone, and slower growth over time. The prevalence of speech or 
language impairments among children is relatively high and comprises a high proportion of children who receive special education services at school entry $(62 \%)^{21}$. This diagnosis has a reciprocal relationship to services for children with learning disability. In kindergarten, $11 \%$ of children with special education services are characterized as having learning disabilities, whereas in $5^{\text {th }}$ grade, the age level for many of the children in this study, $60 \%$ of children receiving special education services are diagnosed with learning disabilities and the percent of children receiving services for speech or language impairment drops to $14 \%^{21}$. The shift seems to be related to a high risk of reading impairment in children with early language impairments, with later diagnosis of reading impairments ${ }^{22}$. The diagnosis of language impairment tends to occur at a younger age than that of reading impairment, and the later reading impairment is identified in academic tasks and targeted as a priority for remediation, even though the language impairments are likely to persist ${ }^{23}$. Children exposed to or living with HIV who have language impairments may be at risk for poor or delayed academic achievement ${ }^{3}$.

Understanding the sources of low performance on language assessments in children with HIV or under HAART treatment for HIV requires differentiation of language impairments associated with cognitive impairments, or as a consequence of hearing impairment. Here we adopt the terminology of Primary impairment (Pri-LI) for children with language impairments with no other known impairments ${ }^{24}$, versus Concurrent impairment, (Con-LI, sometimes referred to as "Secondary LI" ${ }^{24}$ ), for children with language impairment along with possible hearing and/or cognitive impairment ${ }^{25}$. Children with HIV could be at higher risk for these concurrent impairments due to the ongoing or intermittent exposure of the developing brain to chronic immune dysregulation that characterizes HIV infection, or due to HIV treatment or other complications of HIV disease. Alternatively, risk for Pri-LI could be increased as a result of HIV infection or other environmental influences that can impair language acquisition. Comparison of the prevalence and risk factors for LI in children with HIV infection as compared to those perinatally HIV-exposed but HIV-uninfected can clarify whether an increased risk of LI is attributable to HIV infection.

Evaluation of risk factors for language impairment, both Pri-LI and Con-LI, is needed to identify possible contributions of HIV disease, disease severity and treatments, while controlling for demographic and caregiver characteristics ${ }^{26}$. This study evaluated the relationship between language ability and nonverbal cognitive ability, and the prevalence of Pri-LI and Con-LI in children perinatally infected with HIV (HIV+) compared to children exposed and uninfected (HEU), and the relationships of HIV-disease characteristics along with environmental risk factors on language impairment.

\section{METHODS}

\section{Study Population}

This investigation used data collected in the Adolescent Master Protocol (AMP), a component of the Pediatric HIV/AIDS Cohort Study (PHACS). AMP is a prospective cohort study conducted at 15 sites in the United States, including Puerto Rico, designed to evaluate the impact of HIV infection and ART on the development of children and adolescents with perinatal HIV exposure. The study protocol was approved by the institutional review board (IRB) of each participating site; written informed consent was obtained from each child's parent/legal guardian or from older participants as allowed by the local IRB. Written assent was obtained as appropriate. The study opened to enrollment in March 2007.

Children ages 7-16 years born to women with HIV infection were eligible. The study design included both children infected with HIV (HIV+) and children exposed and uninfected (HEU). At each semi-annual study visit, information about study participants and their 
families was gathered through clinical interviews, medical record reviews, and neurodevelopmental testing. The current study is a cross-sectional analysis of language data collected at the first six-month follow up visit, the first time language was assessed. Lifetime health and treatment histories were obtained through chart reviews and current health status was ascertained through physical and laboratory evaluations.

\section{Assessment of Language Impairment}

Language functioning was evaluated using the comprehensive Clinical Evaluation of Language Functioning-Fourth Edition (CELF-4) ${ }^{27}$. Language Impairment (LI) was defined as scoring more than one standard deviation (SD) below the reference mean (CELF-4 Core Language Standard Score less than 85 , equivalent to the $16^{\text {th }}$ percentile). This criterion was motivated by the observation that the norming samples for CELF-4 (reported in the test manual, p. 207) included children from bilingual homes (15\%) as well as children receiving special services (9.5\% overall, $4.8 \%$ for learning disability or intellectual disability, and $7.0 \%$ for speech or language services). Further, the criterion of one standard deviation below the mean is commonly used in studies of children with SLI, allowing comparison across studies $^{28}$.

The age range of the AMP study crosses two versions of the CELF-4 protocol. Children ages 7 and 8 years completed the following subtests: Concepts and Following Directions; Word Structure, Recalling Sentences, and Formulated Sentences. Children ages 9 and older completed these subtests: Concepts and Following Directions, Recalling Sentences, Formulated Sentences, Receptive Word Classes and Expressive Word Classes. The Core Language Standard Score was computed for the age appropriate subtests, yielding a comparable standard score across age levels.

LI was classified as Pri-LI (LI with no cognitive or hearing impairment) or Con-LI (LI with cognitive or hearing impairment). Audiometric hearing examinations or hearing screens were requested according to the study protocol for children who scored in the LI range; $72 \%$ of those with LI received audiometric exams, $7 \%$ received routine hearing screenings, and $21 \%$ had a caregiver report of presence or absence of permanent hearing loss or concerns regarding the child's hearing. In some cases caregiver reports were based on prior audiometric exams. Nonverbal cognitive impairment was defined as a standard score less than 85 , one standard deviation below the mean $(<-1 \mathrm{SD})$, on the Perceptual Reasoning Index (PRI) of the Wechsler Intelligence Scale for Children-Fourth Edition (WISC-IV) ${ }^{29}$. The possible confounding effect of multilingual exposure on LI was considered. For those children administered the CELF-4 in English, multilingual exposure was defined as exposure to non-English language either at home or outside the home. The CELF-4 (as well as the WISC-IV) was given in Spanish to children whose primary language was Spanish, based on parent report and/or examiner judgment. For these children, exposure to other languages was not collected and they were classified as monolingual. For the analyses of Pri-LI and Con-LI, children with LI who also had multilingual exposure could not be classified as Pri-LI; they were classified as Con-LI only if they met the criteria for cognitive or hearing impairment. See further detail in the Results section.

\section{Assessment of Other Risk Factors for Language Impairment}

The ART regimen at the time of CELF administration was determined from medication regimen data collected in AMP. Age at ART initiation was based on the earliest date of ARV use, excluding neonatal prophylaxis in the first two months of life and treatment durations less than a week, determined by review of individual records. HIV viral load and CD4+ T-lymphocyte count and percent, two widely used indicators of HIV disease severity (see guidelines at http://aidsinfo.nih.gov/contentfiles/Pediatric_Guidelines.pdf) were 
obtained as part of routine medical care; results closest to the CELF administration were used in our analysis. Current or past diagnosis with an AIDS-defining condition was based on CDC clinical classification, determined by pediatric surveillance definitions for participants younger than 13 years of age (1994) and the revised adult case definition of AIDS-defining conditions for participants 13 years of age or older (1993).

Factors widely considered as predictors of children's language acquisition were also evaluated as potential confounders in our analysis. Caregiver's education (low level defined as less than high school degree), household income (low level defined as $<\$ 20,000$ annual income), marital status, and relationship to child (biological parent or other) are potential influences on children's development ${ }^{30}$ although environmental prediction of language impairments has yielded mixed outcomes in large population samples ${ }^{31}$. These variables were obtained by caregiver self-report at study entry. Maternal ARV drug use during pregnancy was obtained at entry in PHACS or from previous studies in which the child and/ or mother participated. Caregiver's performance IQ (PIQ) was included as an index of nonverbal cognitive functioning, obtained by direct assessment with the Wechsler Abbreviated Scale of Intelligence, with impairment defined as $<70^{32}$.

\section{Statistical Methods}

The relationship of language and nonverbal intelligence across the full sample was examined based on Pearson correlation coefficients and inspection of bivariate distributions. Children in the HIV+ and HEU groups were compared with respect to demographic and caregiver characteristics. The prevalence of Pri-LI, Con-LI, and no LI was summarized by demographic and caregiver characteristics, and by HIV disease characteristics among the children with HIV. T-tests or ANOVAs and Fisher's Exact tests were used to compare characteristics between HIV+ and HEU groups and across language impairment categories, as appropriate. Univariate and multivariable logistic regression models were used to evaluate the association of HIV status and other factors with the presence of Pri-LI and ConLI, each versus No LI, both overall and within the HIV+ group. Risk factors considered for the HIV+ group also included measures of HIV disease severity. Initial multivariable models included all covariates with $p<0.20$ in univariate models. To be as inclusive as possible, final adjusted models retained all covariates with $p<0.15$. Because of the specific interest in evaluating the association of HIV infection status with LI, this variable was included in all of the models for the overall population.

To provide additional power for detecting differences in language functioning by HIV infection status, sensitivity analyses were conducted by considering the continuous CELF scores and comparing the HIV+ to HEU group using linear regression models adjusted for other confounders. These models were fit with and without child cognitive impairment to address scientific interest in primary versus concurrent impairment. Analyses were conducted using SAS Version 9 (SAS Institute, Cary, NC), and were based on data submitted as of March 1, 2010.

\section{RESULTS}

A total of 468 children ( $306 \mathrm{HIV}+$ and $162 \mathrm{HEU}$ ) had a valid and complete language assessment, at a median age of 12 years (range 7-16); the children were 52\% male, 69\% Black and 26\% Hispanic. Table 1 displays demographic and caregiver characteristics by HIV infection status for the 437 children in whom Pri-LI, Con-LI and no LI could be distinguished (excluding 8 children due to missing WISC-IV PRI scores and 23 children with LI and multilingual exposure but no other concurrent hearing or nonverbal cognitive impairment). The children with HIV in our study were more likely to be older, female, Black, and non-Hispanic than HEU children. While there were no significant differences in 
most caregiver characteristics (education, marital status, or performance IQ) between the HIV+ and HEU groups, children with HIV were less likely to be from a low income household and to have their biological parent as their caregiver. As expected, children with HIV were less likely to have been exposed to ARV drugs in utero, as compared to children in the HEU group (18\% vs. $85 \%)$.

Most children (93\%) were administered the CELF-4 in English. Of the 437 children, 153 (35\%) scored more than one SD below the general population mean on the CELF-4 Core Language Score $(\mathrm{CELF}<85)$. The rates of LI were comparable between the HIV+ and the HEU groups (34\% vs $37 \%$ respectively) and the mean Core Language scores were also similar (88.5 vs 87.5$)$. Overall, 32 children (7\%) had hearing impairment, $88(19 \%)$ had multilingual exposure, and 137 (29\%) had low nonverbal cognitive scores $(<85)$.

Among the 437 children for whom LI category could be identified 48 (11\%) had Pri-LI and 105 (24\%) had Con-LI. Concurrent conditions for the children with Con-LI included a low nonverbal cognitive score for 94 (90\%), hearing impairment for 20 (19\%) and both low nonverbal cognitive score and hearing impairment for nine (9\%). Multilingual exposure was present in combination with either low nonverbal cognitive score or hearing impairment for $18(17 \%)$. Of the children without LI, 43 (15\%) had low nonverbal cognitive score, $12(4 \%)$ had abnormal hearing, and $47(17 \%)$ had multilingual exposure. One child $(<1 \%)$ of the 284 without LI had both a low nonverbal cognitive score and hearing impairment.

The percent within each language impairment category by HIV infection status and demographic factors is summarized in Table 2. The prevalence of Pri-LI and Con-LI were similar in the HIV+ and HEU groups, with $10 \%$ vs $12 \%$ for Pri-LI, and $24 \%$ vs $25 \%$ for Con-LI, respectively. The mean CELF-4 Core Language standard scores were highest for those with no LI (98.7) and slightly higher for those with Pri-LI as compared to Con-LI (74.2 vs 65.8). The Pearson correlation between nonverbal IQ and CELF Core Language Standard Scores was 0.63 among all subjects, but higher for those with Con-LI (0.51) and those with no language impairment (0.37) than for children with Pri LI (0.29). Most children had consistent levels of nonverbal IQ and language functioning, with 54\% scoring above 85 for both the nonverbal WISC and CELF-4 and 24\% scoring below 85 for both (i.e., Con-LI). However, 91 children (21\%) had inconsistent measures of functioning, with $11 \%$ showing only language deficits and $10 \%$ observed with normal language functioning but low nonverbal IQ. These inconsistencies in the general continuum of severity motivate separate evaluation of predictor relationships for Con-LI and Pri-LI, to examine possible differences between the two groups of children with LI.

A summary of HIV disease severity by language impairment group is provided in Table 3 and illustrated in Figure 1. Children with Con-LI were significantly more likely than those with Pri-LI or no LI to have initiated ART by 6 months of age, to have a prior CDC Class C diagnosis, a detectable viral load, and CD4\% $<25 \%$. Most children were on HAART with protease inhibitor (PI) at the six-month visit $(72 \%)$ with no differences in ART regimen by LI group.

Table 4 summarizes the results of univariate and final adjusted multivariable logistical regression models for Pri-LI vs no impairment, both overall (upper panel) and within the group of children with HIV (lower panel). For the overall study population, the final adjusted model indicated no significant difference in the odds of Pri-LI vs No LI for HIV+ as compared to HEU groups of children; however, children who were Black had over twice the odds of Primary LI vs. no LI ( $\mathrm{aOR}=2.43, p=0.03)$.

Table 5 presents the results of analogous models for Con-LI vs no LI groups, again both overall (upper panel) and within the group of children with HIV (lower panel). Univariate 
logistic models for Con-LI versus No LI among HIV + and HEU revealed a significant effect of caregiver education ( $p=0.01$ ) and a marginal effect of low caregiver nonverbal IQ $(p=0.06)$, but no significant association with HIV status or other characteristics. However, in the final adjusted model, a significant increase in the odds of Con-LI was observed for children who were Black $(\mathrm{aOR}=3.7, p=0.003)$ or Hispanic $(\mathrm{aOR}=3.2, p=0.01)$, who had a less educated caregiver $(\mathrm{aOR}=1.8, p=0.04)$ or a caregiver with low cognitive score $(\mathrm{aOR}=3.3, p=0.05)$ or had a caregiver who was not the biological parent $(\mathrm{aOR}=1.9, p=0.02)$. There was no significant effect of HIV infection status on odds of Con-LI vs no LI.

In a sensitivity analysis based on the continuous CELF-4 scores reported in Table 6, linear regression models indicated no difference in adjusted mean scores between HIV+ vs HEU ( mean difference $=0.44,95 \% \mathrm{CI}:-3.20,4.08, \mathrm{p}=0.81$ ). However, participants of Black race had an adjusted mean CELF-4 score which was 6.3 points lower than those of non-Black race $(\mathrm{p}=0.002)$, and those of lower socioeconomic status had lower mean scores, as reflected by having a caregiver without high school education (4.9 points lower, $\mathrm{p}=0.02)$ and an annual household income below $\$ 20,000$ (3.4 points lower, $\mathrm{p}=0.06$ ). In addition, low caregiver cognitive score based on PIQ $<70$ was associated with significantly lower adjusted mean scores (10.0 points lower, $\mathrm{p}=0.04$ ). Further adjustment for child's cognitive level led to slight attenuation of differences in adjusted means noted above, but race, caregiver education, and low caregiver nonverbal IQ remained significantly associated with CELF-4 scores while HIV status remained clearly non-significant (mean difference $=0.8, \mathrm{p}=0.62$ ).

Within the HIV+ group, univariate and adjusted logistic regression models investigating disease severity measures are summarized in Table 4 (bottom panel) for Pri-LI vs no LI and Table 5 (bottom panel) for Con-LI vs no LI. In univariate models, none of the disease measures reached statistical significance for predicting the odds of Pri-LI vs no LI. In the multivariate model, only age at ART initiation reached significance indicating a two-fold increase in odds of Pri-LI for those with later ART initiation. In contrast, those with detectable viral load, prior CDC Class C diagnosis, or earlier ART initiation had two to three-fold higher odds of Con-LI vs no LI. Within the HIV+ subgroup, children who were male or had a biological parent as caregiver had significantly lower odds of Con-LI, while those who were Black or had a caregiver with lower education had significantly higher odds. Although children with HIV with lower CD4\% $(<25 \%)$ had significantly increased odds of Con-LI in univariate models, this association did not persist after adjustment for other measures of HIV disease severity. Similarly, in sensitivity analyses based on linear regression models for the continuous CELF-4 score restricted to participants with HIV, only HIV viral load $>400$ copies $/ \mathrm{mL}$ and CDC Class $\mathrm{C}$ were associated with lower mean CELF scores (5.8 and 4.3 points lower, respectively). While children with low CD4\% had marginally lower mean scores in unadjusted models, this finding did not persist after adjustment for viral load and CDC Class. After further adjustment for child's nonverbal IQ, CDC Class $\mathrm{C}$ was no longer associated with CELF scores, but child's nonverbal IQ was associated with a 19.1 point lower adjusted mean CELF-4-4 score (see Table 6).

\section{DISCUSSION}

Language impairment in this cohort was common among children in both HIV+ and HEU groups, with impairment observed among almost $40 \%$ of participants as compared to an expected rate of $16 \%$ in the US population ${ }^{27}$. The elevated risk is especially noteworthy given that recent studies note that the practice of including children with language impairments in the norming data of tests such as CELF-4 reduces test sensitivity for identifying children with language impairments ${ }^{33}$. The rate of LI was similar for children in HIV+ and HEU groups, both overall and within sub-classifications of Pri-LI or Con-LI. The rates of LI in this study are higher than the rates of LI reported in other studies. For example, 
the overall prevalence of SLI, psychometrically defined similarly to Pri-LI, was $7.4 \%$ in a study of healthy five- to six-year-old children ${ }^{18}$. Prevalence of SLI for urban AfricanAmerican children and for primarily urban Hispanic children (both groups were primarily low SES) in that study was $11 \%$ and $8 \%$, respectively. An additional $5 \%$ of the cohort demonstrated low nonverbal cognitive functioning accompanied by language impairment, while $12 \%$ performed at low nonverbal cognitive levels (70-87 IQ) without language impairment ${ }^{19}$. In our study, we found that $10 \%$ of the children without LI had low nonverbal cognitive scores $(<85)$. Low nonverbal cognitive abilities thus could occur with or without accompanying language impairment and vice-versa.

Results from the logistic regression models indicate that factors predictive of LI differ for Pri-LI and Con-LI, yet risk of both types of LI was not different for children in HIV+ and HEU groups. The risk factors examined in these analyses revealed very little about the sources of risk for Pri-LI in children perinatally exposed to HIV. Other studies report genetic linkage and association for SLI ${ }^{28,34-35}$, suggesting that genetic influences may contribute to risk in the children exposed to HIV. In the HIV+ and HEU groups of children in this sample, the established demographic and psychosocial risk factors for Con-LI were notably elevated relative to reference norms. For the HIV+ group, markers of greater disease severity and poorer current control were additional risk factors for Con-LI, suggesting that the HIV disease process may produce more global impairments as seen in Con-LI; the lack of association of these measures with Pri-LI suggests that advanced HIV disease is less likely to cause language impairment in isolation. It is notable that 25 of the 59 children with CDC Class C diagnoses had prior reported diagnoses of encephalopathy ${ }^{36-37}$. It is also possible that the smaller number of children with Pri-LI reduced power to detect associations; however, a sensitivity analysis for the continuous CELF-4 scores offered some confirmation of this finding in that few HIV disease severity measures were associated with mean CELF-4 scores after adjustment for children's low nonverbal cognitive status, akin to lower mean CELF-4 scores not explained by children's cognitive status. With approximately 275 $\mathrm{HIV}+$ and $150 \mathrm{HEU}$ subjects, the linear regression approach had $80 \%$ power to detect differences of 4.3 points or more in mean CELF- 4 scores.

Age at initiation of ART was associated with both Pri-LI and Con-LI but the effect was in opposite directions. Early ART was associated with higher odds of Con-LI, but significantly lower odds of Pri-LI. The negative effects of HIV infection on the central nervous system can occur prior to birth and during infancy ${ }^{38}$, before many of the participants in this study initiated ART. Though current guidelines recommend routine treatment from early infancy, our study population consisted primarily of older children born at a time when they would have more likely initiated ART early if they manifested neurologic impairment and other serious HIV-related disease in infancy. Thus, ART initiation before 6 months of age may be associated with Con-LI because it is a marker for those children who experienced more severe neurologic involvement from an early age. Among children who did not exhibit overt neurologic problems in infancy, subclinical brain involvement was nonetheless likely. For these children, early ART may have limited or prevented development of neurodevelopmental problems like Pri-LI, while later initiation of ART ensured survival but may have permitted a longer pre-treatment period of HIV-related effects on brain function that placed them at higher risk for more subtle or focused problems, such as Pri-LI. On the other hand, early ART in the current study may reflect early and perhaps more consistent access to comprehensive medical and psychological care and support, including referrals to appropriate early intervention and special education services if delays or deficits are recognized early. There may also be uncontrolled confounding by disease, treatment or temporal factors associated with age at ART initiation that underlie these associations, but none were evident in the current analysis. Early identification of HIV infection with routine 
initiation of HAART in young infants in the United States, as currently recommended and practiced, may result in different language outcomes in future cohorts.

We did not identify an association between current ART with increased risk of either Primary or Concurrent LI among youth with perinatal HIV. However, our ability to detect associations may have been limited by the relatively small percentage of youth not receiving HAART. Additionally, the majority of children were fairly HAART-experienced ( $86 \%$ had over 5 years of HAART) which limited our ability to distinguish language functioning by duration on HAART. Despite widespread HAART use in our study population, $46 \%$ of those with Con-LI had viral load $>400$ copies $/ \mathrm{mL}$, suggesting adherence difficulties or the possibility of drug resistance. Detectable viral load at the time of language testing was associated with significantly higher odds of language impairment concurrent with hearing and/or low nonverbal cognitive scores. This finding suggests that effectiveness of HAART in reducing deficits in language functioning observed in the pre-HAART era may depend on careful monitoring of both adherence and resistance patterns to guide optimal therapy.

There were some limitations of our study. Our analysis was a cross-sectional evaluation of language functioning at a single time point. Future longitudinal follow-up of these children is necessary to determine the persistence of Pri-LI and Con-LI and possible long-term risks associated with disease status, treatment, and other risk factors. In addition, despite attempts to enroll a comparable control group of uninfected children, the HEU children tended to be younger and living in lower income households. While adjustment for these factors addressed these imbalances to the extent possible, this illustrates the difficulty of identifying a comparable control group of children with perinatal HIV exposure. In addition, we were unable to obtain formal audiometric exams on all children with hearing impairment. Nevertheless, to our knowledge, this is the first study which specifically addresses hearing impairment in evaluation of language functioning among children with HIV. The major strengths of this study include the focus on language impairment, particularly with respect to its classification into Primary and Concurrent groups, the relatively large sample size, the use of a group of HEU children for comparison, and the ability to control for potential confounders.

Several conclusions apply to clinical practice. Physicians providing primary and specialty care to children perinatally exposed to HIV need a heightened awareness of the high rates of LI among children of mothers with HIV, interactions between the disease and its treatment, and routine preventive screening, including hearing assessments. Although Con-LI may be more salient and readily ascertained, children without cognitive problems with HIV infection and children exposed but uninfected experience Pri-LI that may be equally impairing but less easily recognized. Ongoing studies of LI are needed for children with HIV as temporal changes in antiretroviral therapy may lead to different patterns of LI in the future. Those providing care to children and youth growing up with perinatal HIV infection or exposure to HIV should be aware of their substantial risk of LI and potential for difficulties in school and careers that such impairments may produce. Early and ongoing intervention could reduce the negative impact of language impairment.

\section{Acknowledgments}

We thank the children and families for their participation in PHACS, and the individuals and institutions involved in the conduct of PHACS.

The following institutions, clinical site investigators and staff participated in conducting PHACS AMP in 2010, in alphabetical order: Baylor College of Medicine: William Shearer, Norma Cooper, Lynette Harris; Bronx Lebanon Hospital Center: Murli Purswani, Mahboobullah Baig, Anna Cintron; Children's Diagnostic \& Treatment Center: Ana Puga, Sandra Navarro, Doyle Patton; Children's Hospital, Boston: Sandra Burchett, Nancy Karthas, Betsy Kammerer; Children's Memorial Hospital: Ram Yogev, Kathleen Malee, Scott Hunter, Eric Cagwin; 
Jacobi Medical Center: Andrew Wiznia, Marlene Burey, Molly Nozyce; St. Christopher's Hospital for Children: Janet Chen, Elizabeth Gobs, Mitzie Grant; St. Jude Children's Research Hospital: Katherine Knapp, Kim Allison, Patricia Garvie; San Juan Hospital/Department of Pediatrics: Midnela Acevedo-Flores, Heida Rios, Vivian Olivera; Tulane University Health Sciences Center: Margarita Silio, Cheryl Borne, Medea Jones; University of California, San Diego: Stephen Spector, Kim Norris, Sharon Nichols; University of Colorado Denver Health Sciences Center: Elizabeth McFarland, Emily Barr, Robin McEvoy; University of Maryland, Baltimore: Douglas Watson, Nicole Messenger, Rose Belanger; University of Medicine and Dentistry of New Jersey: Arry Dieudonne, Linda Bettica, Susan Adubato; University of Miami: Gwendolyn Scott, Lisa Himic, Elizabeth Willen

Funding: The study was supported by the Eunice Kennedy Shriver National Institute of Child Health and Human Development with co-funding from the National Institute on Drug Abuse, the National Institute of Allergy and Infectious Diseases, the National Institute of Mental Health, the National Institute of Neurological Disorders and Stroke, the National Institute of Deafness and Other Communication Disorders, the National Heart Lung and Blood Institute, and the National Institute on Alcohol Abuse and Alcoholism through cooperative agreements with the Harvard University School of Public Health (HD052102) (Principal Investigator: George Seage; Project Director: Julie Alperen) and the Tulane University School of Medicine (HD052104) (Principal Investigator: Russell Van Dyke; Co-Principal Investigator: Kenneth Rich; Project Director: Patrick Davis). Data management services were provided by Frontier Science and Technology Research Foundation (PI: Suzanne Siminski), and regulatory services and logistical support were provided by Westat, Inc (PI: Julie Davidson).

Note: The conclusions and opinions expressed in this article are those of the authors and do not necessarily reflect those of the National Institutes of Health or U.S. Department of Health and Human Services.

Disclosure: Funded by the National Institutes of Health

\section{REFERENCES}

1. Blanchette N, Smith ML, King S, Fernandes-Penney A, Read S. Cognitive development in schoolage children with vertically transmitted HIV infection. Dev. Neuropsychol. 2002; 21(3):223-241. [PubMed: 12233936]

2. Martin SC, Wolters PL, Toledo-Tamula MA, Zeichner SL, Hazra R, Civitello L. Cognitive functioning in school-aged children with vertically acquired HIV infection being treated with highly active antiretroviral therapy (HAART). Dev. Neuropsychol. 2006; 30(2):633-657. [PubMed: 16995830]

3. Brackis-Cott E, Kang E, Dolezal C, Abrams EJ, Mellins CA. The impact of perinatal HIV infection on older school-aged children's and adolescents' receptive language and word recognition skills. AIDS Patient Care STDS. 2009; 23(6):415-421. [PubMed: 19415986]

4. Brackis-Cott E, Kang E, Dolezal C, Abrams EJ, Mellins CA. Brief Report: Language ability and school functioning of youth perinatally infected with HIV. J. Pediatr. Health Care. 2009; 23(3):158164. [PubMed: 19401248]

5. Coplan J, Contello KA, Cunningham CK, et al. Early language development in children exposed to or infected with Human Immunodeficiency Virus. Pediatrics. 1998; 102(1):e8. [PubMed: 9651460]

6. Malee K, Williams P, Montepiedra G, et al. The role of cognitive functioning in medication adherence of children and adolescents with HIV infection. J. Pediatr. Psychol. 2009; 34:164-175. [PubMed: 18647794]

7. Smith R, Malee K, Leighty R, et al. Effects of perinatal HIV infection and associated risk factors on cognitive development among young children. Pediatrics. 2006; 117:851-862. [PubMed: 16510667]

8. Wolters PL, Brouwers P, Civitello L, Moss HA. Receptive and expressive language function of children with symptomatic HIV infection and relationship with disease parameters: a longitudinal 24-month follow-up study. AIDS. 1997; 11:1135-1144. [PubMed: 9233461]

9. Hopkins K, Grosz J, Cohen H, Diamond G, Nozyce M. Emerging patterns of services and case finding for children with HIV infection. Ment. Retard. 1989; 27:219. [PubMed: 2479809]

10. Raskino C, Pearson DA, Baker CJ, et al. Neurologic, neurocognitive, and brain growth outcomes in human immunodeficiency virus-infected children receiving different nucleoside antiretroviral regimens. Pediatrics. 1999; 104:e32. [PubMed: 10469815]

11. Lindsey JC, Malee KM, Brouwers P, Hughes MD. Team PCS. Neurodevelopmental functioning in HIV-infected infants and young children before and after the introduction of protease inhibitorbased highly active antiretroviral therapy. Pediatrics. 2007; 119:e681-e693. [PubMed: 17296781] 
12. Jeremy RJ, Kim S, Nozyce M, et al. Neuropsychological functioning and viral load in stable antiretroviral therapy-experienced HIV-infected children. Pediatrics. 2005; 115:380-387. [PubMed: 15687448]

13. Tepper VJ, Farley JJ, Rothman MI, et al. Neurodevelopmental/neuroradiologic recovery of a child infected with HIV after treatment with combination antiretroviral therapy using the HIV-specific protease inhibitor Ritonavir. Pediatrics. 1998; 101:e7. [PubMed: 9493492]

14. Chiriboga C, Fleishman S, Champion S, Gaye-Robinson L, Abrams E. Incidence and prevalence of HIV encephalopathy in children with HIV infection receiving highly active anti-retroviral therapy (HAART). The Journal of Pediatrics. 2005; 146(3):402-407. [PubMed: 15756229]

15. Crain MJ, Chernoff MC, Oleske JM, et al. Possible mitochondrial dysfunction and its association with antiretroviral therapy use in children perinatally infected with HIV. The Journal of Infectious Diseases. 2010; 11:291-301. [PubMed: 20533872]

16. Fischel-Ghodsian N. Mitochondrial deafness mutations reviewed. Hum. Mutat. 1999; 13:261-270. [PubMed: 10220138]

17. Zhao H, Young W, Yan Q, et al. Functional characterization of the mitochondrial 12S rRNA C1494T mutation associated with aminoglycoside-induced and non-syndromic hearing loss. Nucleic Acids Res. 2005; 33(3):1132-1139. [PubMed: 15722487]

18. Tomblin JB, Records NL, Buckwalter P, Zhang X, Smith E, O'Brien M. The prevalence of specific language impairment in kindergarten children. J. Speech Hear. Res. 1997; 40:1245-1260.

19. Shriberg LD, Tomblin JB, McSweeny JL. Prevalence of speech delay in 6-year-old children and comorbidity with language impairment. J. Speech Lang. Hear. Res. 1999; 42:1461-1481.

[PubMed: 10599627]

20. Rice ML, Tomblin JB, Hoffman L, Richman WA, Marquis J. Grammatical tense deficits in children with SLI and nonspecific language impairment: Relationships with nonverbal IQ over time. J. Speech Lang. Hear. Res. 2004; 47:816-834. [PubMed: 15324288]

21. Mashburn AJ, Myers SS. Advancing research on children with speech-language impairment: An introduction to the early childhood longitudinal study-Kindergarten cohort. Language, Speech, and Hearing Services in Schools. 2010; 41:61-69.

22. Catts HW, Bridges MS, Little TD, Tomblin JB. Reading achievement growth in children with language impairments. Journal of Speech, Language, and Hearing Research. 2008; 51:1659-1579.

23. Johnson C, Beitchman JH, Young A, et al. Fourteen-year follow-up of children with and without speech/language impairments: Speech/language stability and outcomes. J. Speech Lang. Hear. Res. 1999; 42:744-760. [PubMed: 10391637]

24. Nelson, NW. Language and Literacy Disorders in Children: Infancy through Adolescence. Boston, MA: Allyn \& Bacon; 2010.

25. Rice ML, Warren SF, Betz SK. Language symptoms of developmental language disorders: An overview of autism, Down syndrome, fragile X, specific language impairment, and Williams syndrome. Applied Psycholinguistics. 2005; 26:7-27.

26. Dollaghan CA, Campbell TF, Paradise JL, et al. Maternal education and measures of early speech and language. J. Speech Lang. Hear. Res. 1999; 42(6):1432-1443. [PubMed: 10599625]

27. Semel, E.; Wiig, EH.; Secord, WA. Clinical Evaluation of Language Fundamentals - 4th edition. 4th ed.. San Antonio, Texas: The Psychological Corporation; 2003.

28. Rice ML, Smith SD, Gayán J. Convergent genetic linkage and associations to language, speech and reading measures in families of probands with Specific Language Impairment. J. Neurodev. Disord. 2009; 1:264-282. [PubMed: 19997522]

29. Wechsler, D. Wechsler Intelligence Scale for Children. San Antonio, TX: the Psychological Corporation; 2003.

30. Entwisle DR, Astone NM. Some practical guidelines for measuring youths' race/ethnicity and socioeconomic status. Child Dev. 1994; 65(6):1521-1540.

31. Zubrick SR, Taylor CL, Rice ML, Slegers D. Late language emergence at 24 months: An epidemiological study of prevalence, predictors, and covariates. J. Speech Lang. Hear. Res. 2007; 50:1562-1592. [PubMed: 18055773]

32. Wechsler, D. Wechsler Abbreviated Scale of Intelligence. San Antonio, TX: The Psychological Corporation; 1999. 
33. Pena ED, Spaulding TJ, Plante E. The composition of normative groups and diagnostic decision making: Shooting ourselves in the foot. American Journal of Speech-Language Pathology. 2006; 15:247-254. [PubMed: 16896174]

34. Falcaro M, Pickles A, Newbury DF, et al. Genetics and phenotypic effects of phonological shortterm memory and grammatical morphology in specific language impairment. Genes Brain Behav. 2008; 7:393-402. [PubMed: 18005161]

35. Vernes SC, Newbury DF, Abrahams BS, et al. A functional genetic link between distinct developmental language disorders. N. Engl. J. Med. 2008. 2008; 359(22):2337-2345.

36. Baraff LJ, Lee SI, Schriger DL. Outcomes of bacterial meningitis in children: A meta-analysis. Pediatr. Infect. Dis. J. 1993; 12(5):389-394. [PubMed: 8327300]

37. Buckingham SC, McCullers JA, Lujan-Zilbermann J, Knapp KM, Orman KL, English BK. Early vancomycin therapy and adverse outcomes in children with pneumococcal meningitis. Pediatrics. 2006; 117(5):1688-1694. [PubMed: 16651325]

38. Chase C, Ware J, Hittelman J, et al. Early cognitive and motor development among infants born to women infected with Human Immunodeficiency Virus. Pediatrics. 2000; 106:e25. [PubMed: 10920181] 


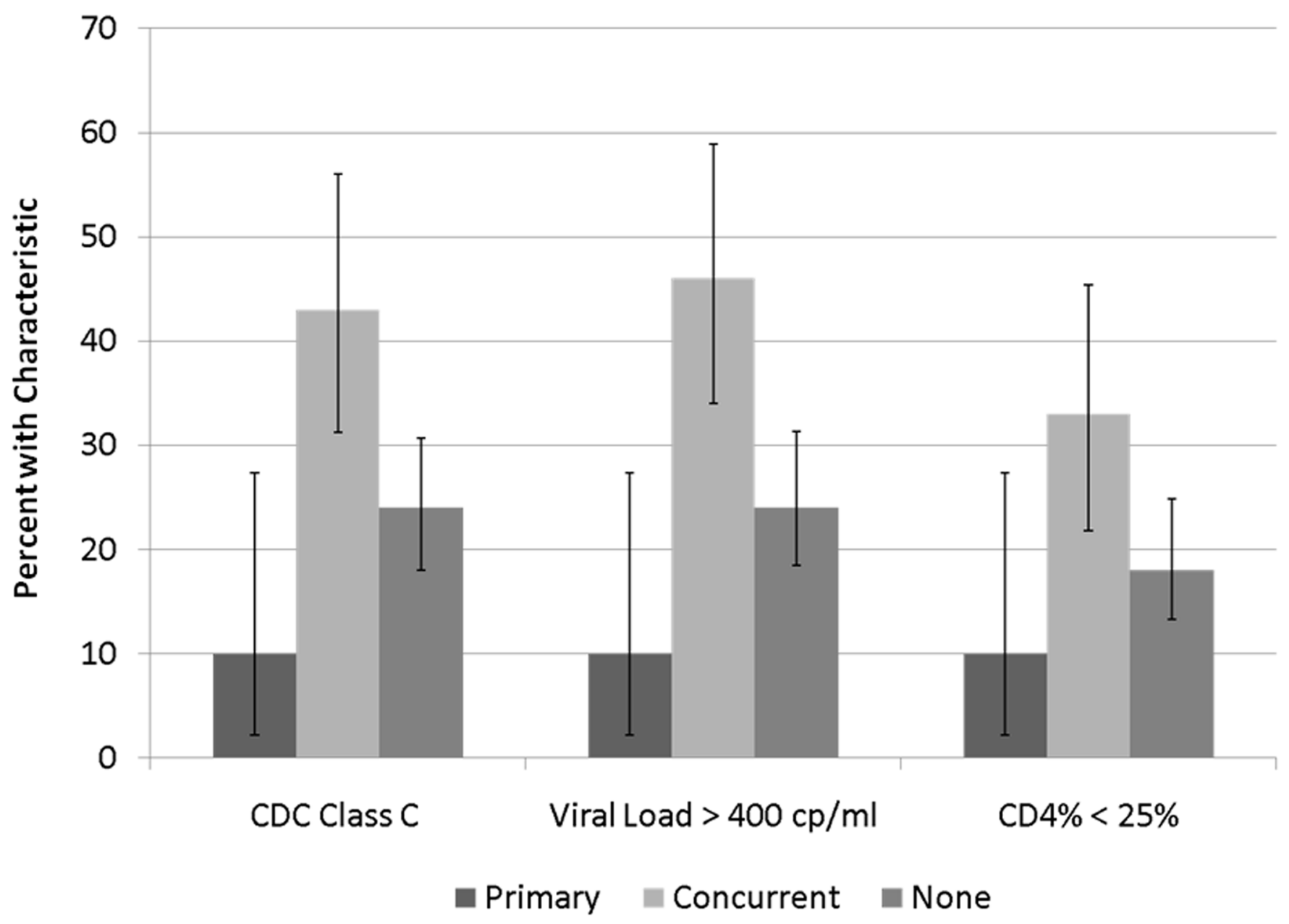

Figure 1.

Percent with Certain Indicators of HIV Disease Severity among Perinatally HIV-infected Children within each Language Impairment Category, with 95\% Confidence Intervals. 


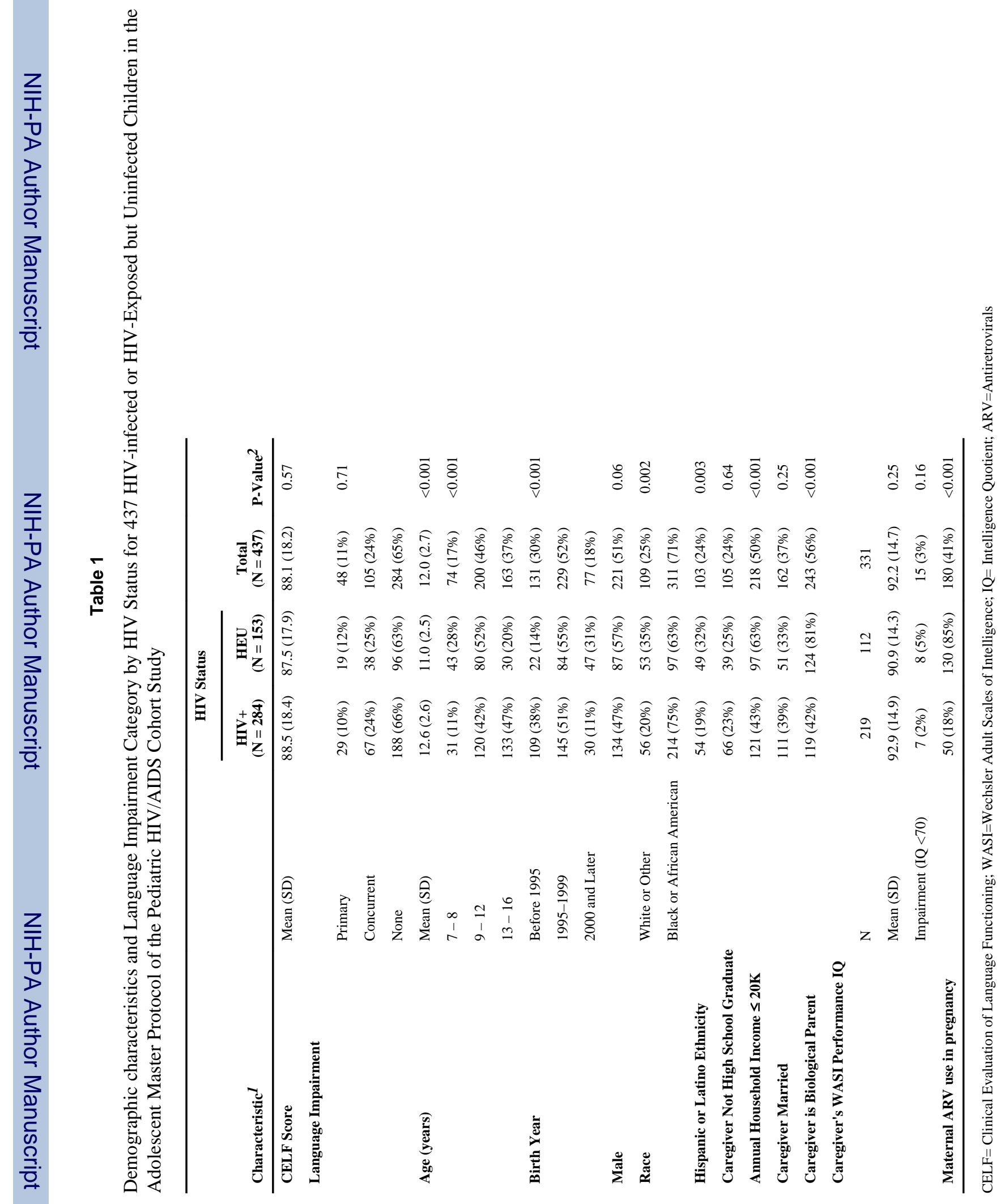




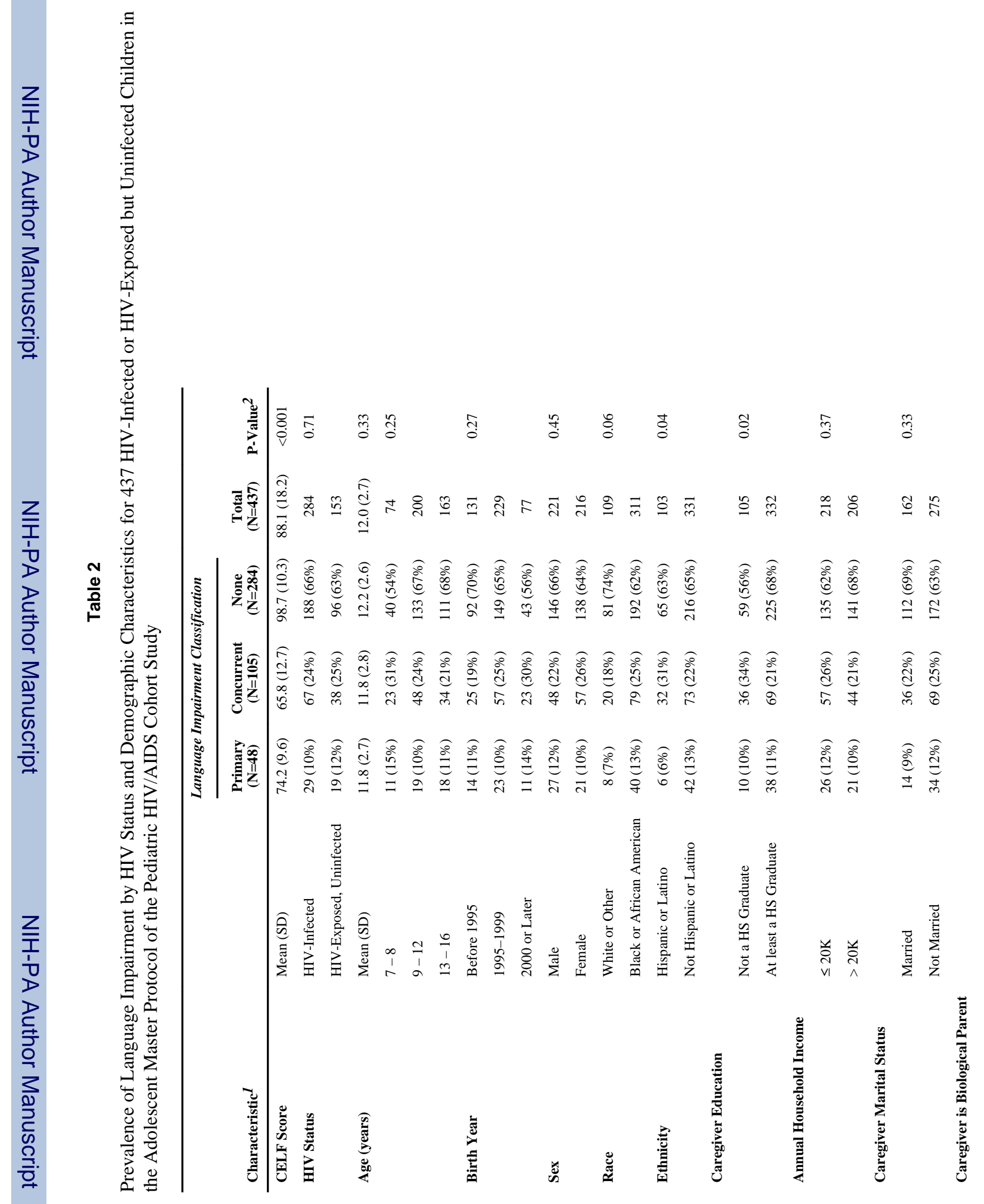




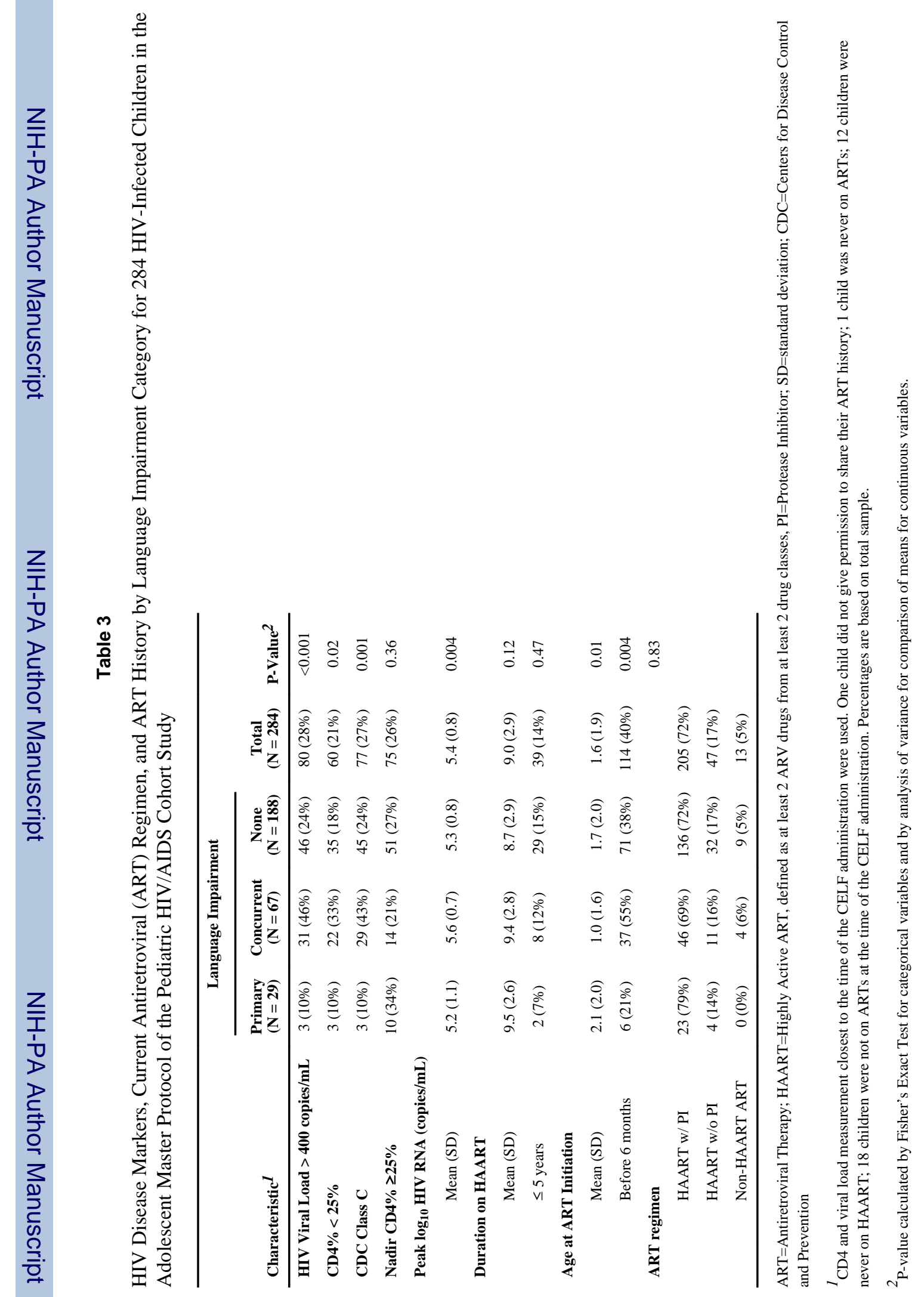




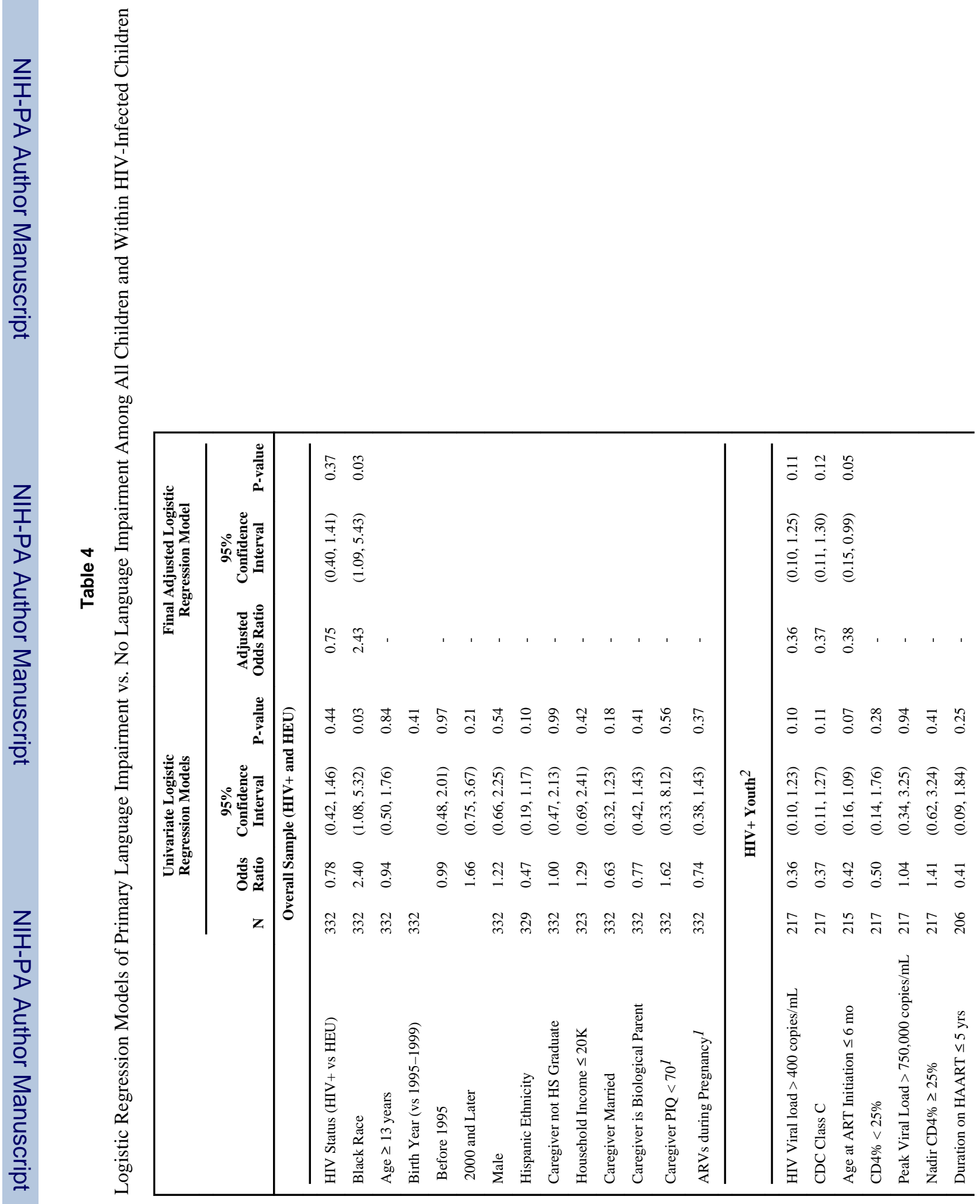




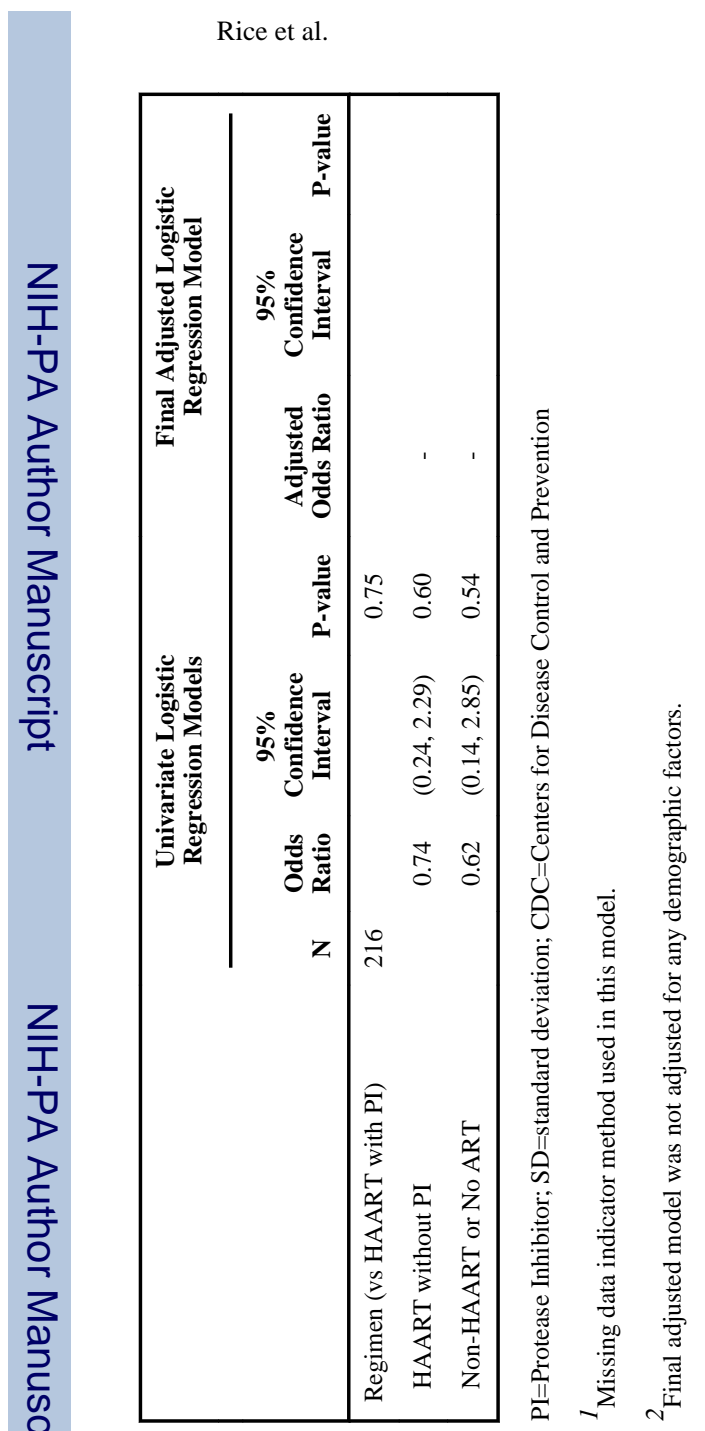

Page 20 


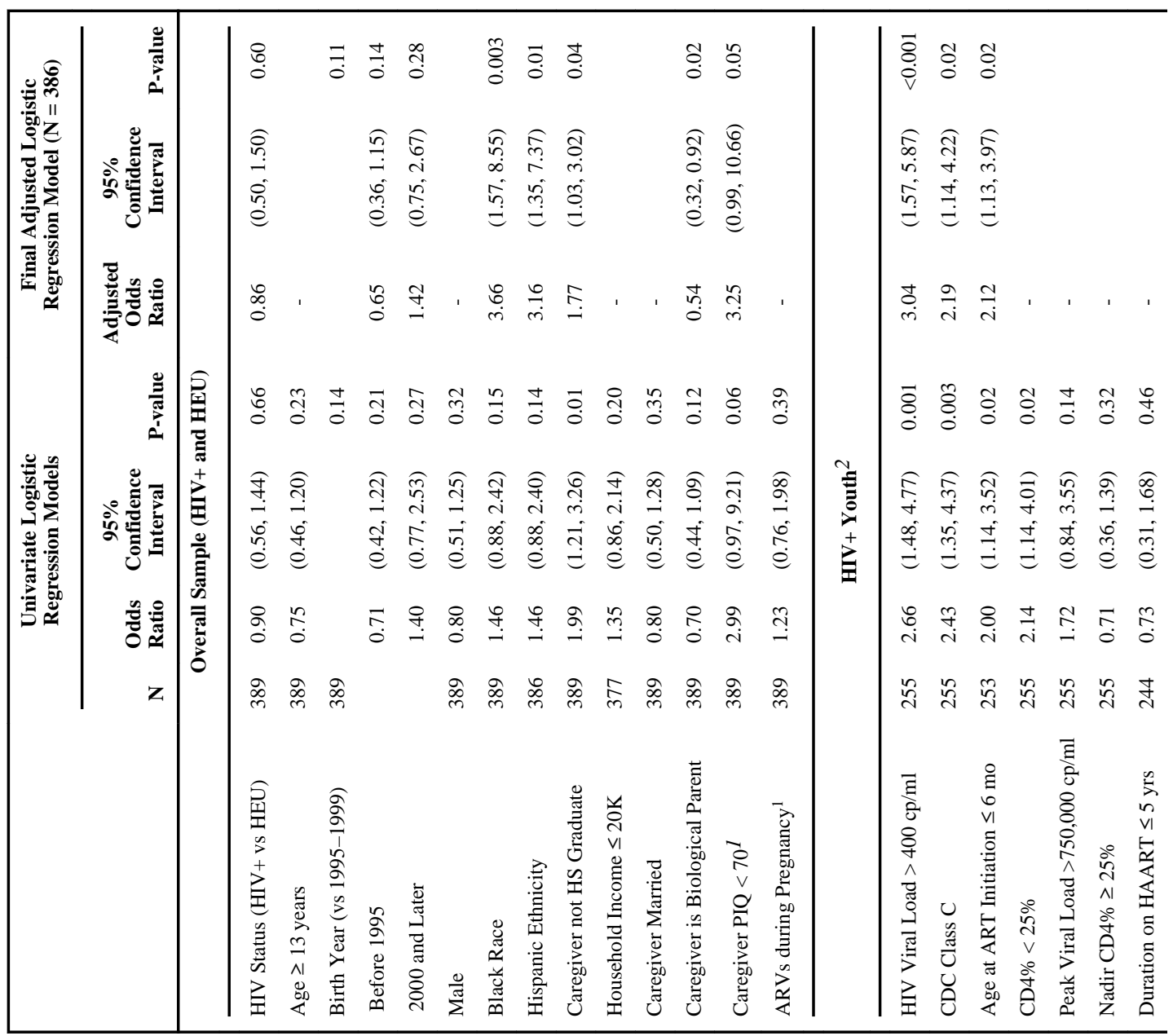




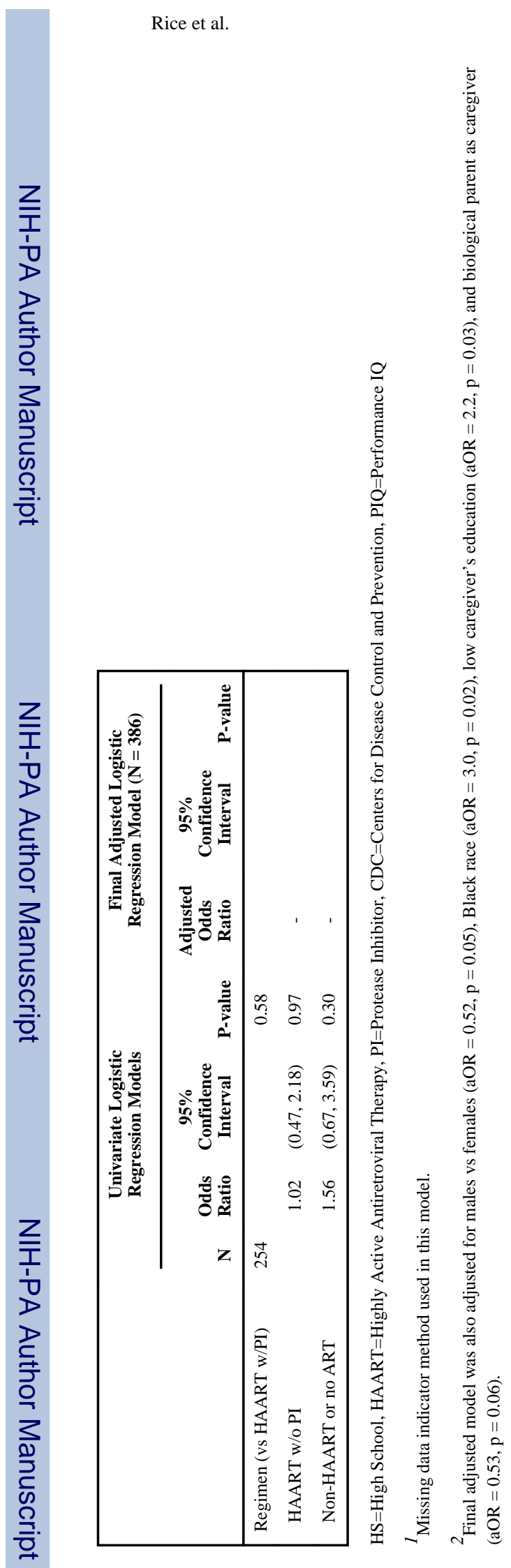

J Dev Behav Pediatr. Author manuscript; available in PMC 2013 February 1. 


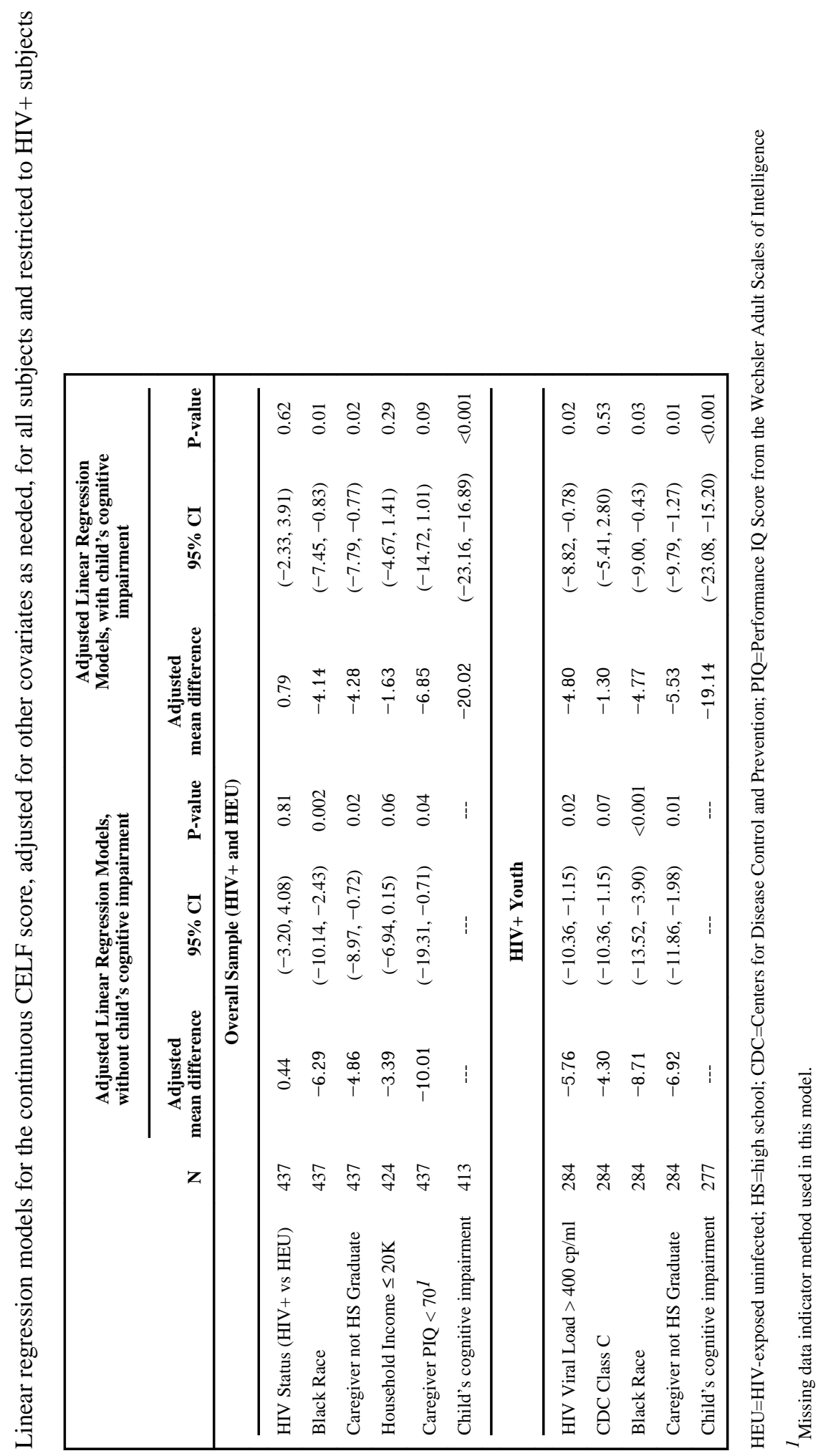

J Dev Behav Pediatr. Author manuscript; available in PMC 2013 February 1. 